\title{
A CP-net Approach To Control Logic Engineering
}

\author{
Mathew Farrington and Jonathan Billington \\ Telecommunications Systems Engineering Centre, \\ Institute for Telecommunications Research, \\ The University of South Australia.
}

\begin{abstract}
Coloured Petri nets have been used to model and therefore specify the control logic in an automotive assembly line. This paper describes the modelling activity and shows how two models of the same system, one developed using top-down methods, the other developed using bottom-up methods, may be fused.
\end{abstract}

\section{Keywords}

Coloured Petri nets, Programmable Logic Controllers (PLCs), Discrete Event Manufacturing, Modelling.

\section{INTRODUCTION}

An automotive assembly line has been analysed and modelled in previous work. The models were developed using the $\mathrm{C}$ programming language and describe the control logic and physical operation of the line. A formal toolkit based on Petri nets is now being used to construct a model of the assembly line from the existing code. The model may then be analysed and verified ensuring that any errors in the assembly line design are uncovered and removed. The resulting CP-net model formally specifies a set of abstract system requirements against which new, distributed system models may be constructed and verified.

This paper describes the initial CP-net modelling activity in which two models were constructed, one from the top down, the other from the bottom up. Knowledge of overall system behaviour was used to construct the top-down model. At each level, agent behavioural requirements and product processing requirements were identified and modelled. The requirements placed on agent, product and process elements implied a more detailed, lower-level model, also consisting of agent, product and process elements. This modelling activity recursed downwards until all the behavioural and processing requirements had been resolved into their discrete control logic and machine-level events. The bottom-up model was constructed by directly representing the ladder logic and physical relationships (actuator and sensor relationships) present in the PLC/system feedback loop using CP-net structures. The final model is a fusion of these two. 


\section{TOOLS AND TECHNIQUES}

Embedded manufacturing control software such as PLC logic is notoriously difficult to maintain since it represents control requirements at a very low level [Gilles, 1990]. Typically this is overcome by specifying and maintaining the code at a higher level where the control relationships are preserved in a more re-usable form [Venkatesh, et al., 1994]. High level code is also more transportable because it is (in theory) platform or hardware independent and may be compiled into PLC ladder logic as required.

Even more promising though are formal methods. They are gaining popularity in the manufacturing control area and have the potential to achieve time and cost savings throughout the life-cycle of a system [Billington, 1991]. If a manufacturing system is designed using formal methods then specification errors are reduced (a maintenance saving); the specification may be executed to quickly gain an understanding of the system requirements (a time saving); the performance of the system may be analysed and verified; the specification may be compiled into code (thus reducing coding errors as well as lowering the time to prototype) - all of this before committing to an implementation.

The Telecommunication Systems Engineering Centre* supports a CP-net toolkit known as FORESEE [Billington, 1991]. It consists of drawing, simulation, formal analysis and implementation tools. The toolkit includes: Design/ $\mathrm{CPN}^{\dagger}$, a graphical editing, simulation and occurrence graph analysis tool; TORAS, a tool for the exhaustive simulation of Petri net specifications using state space reduction techniques [Lester, 1994]; and PROMPT a tool for the automatic conversion of Petri nets into executable code (C).

\section{AN INTRODUCTION TO CP-NETS}

\subsection{Assembly Line Overview}

A brief introduction to the assembly line is needed before the Coloured Petri net models are introduced. The automotive assembly line is divided into stages or work cells each with its own set of actuators, sensors, welding stations, conveyors etc. Each stage assembles components or attaches components to a vehicle as it progresses through the line. One PLC controls the entire system. The system is only partially automated. Workers must perform positioning, loading and spot welding tasks. Worker safety is an integral feature of the system control logic. For example, a conveyor will advance only when both workers in a two person work cell are at their control consoles.

A sub-section of the assembly line, known as the roofing bay, accepts a bare chassis, attaches reinforcing struts and roof panels and then releases the chassis to the next bay. The roofing bay is the focus of this paper.

\footnotetext{
*The TSEC web page, http://www.itr.unisa.edu.au/tsec/.

† Design/CPN is available from Aarhus University, http://www.daimi.aau.dk/designCPN/ .
} 


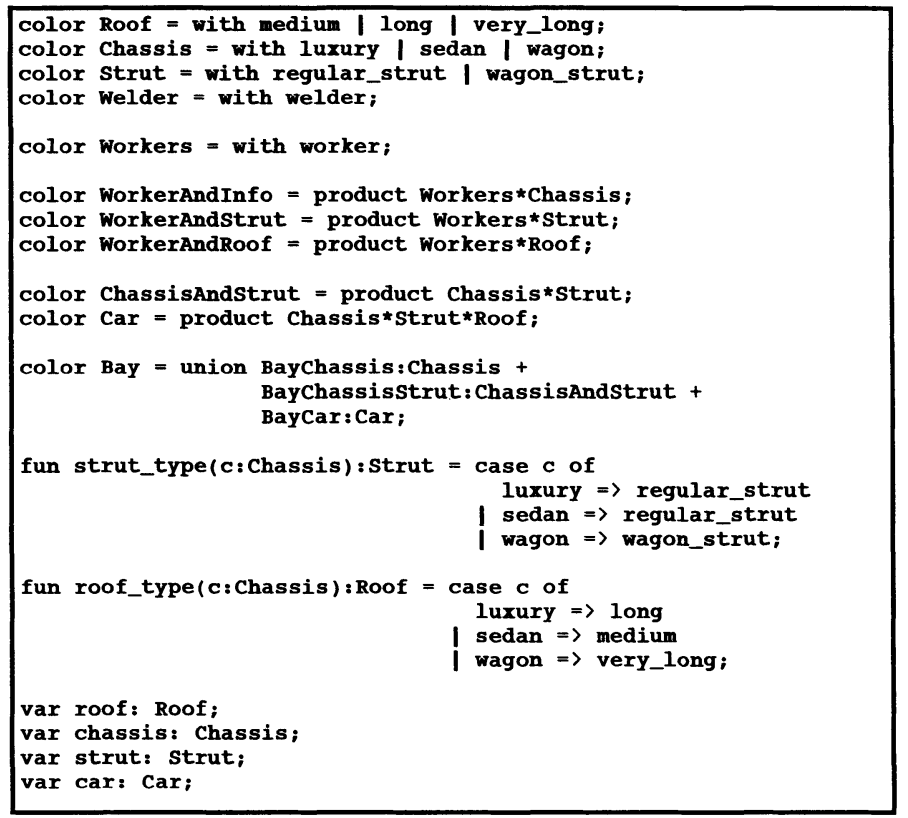

Figure 1 Global Declaration Page for the Assembly Line

\subsection{CP-nets by Example}

It is assumed that the reader is familiar with basic Petri net concepts [Reisig, 1992]. Coloured Petri nets (CP-nets) are essentially ordinary Petri nets (Place Transition nets) with typed tokens and complex net inscriptions [Jensen, 1992]. CP-nets can specify and model systems at an implementation independent level. CP-nets are based on a well defined semantics - a system specified in CP-nets may be formally analysed or verified before implementation. CP-nets can model concurrent processes and are therefore naturally suited to the design and modelling of concurrent manufacturing systems. In this short introduction to $\mathrm{CP}$-nets their semantics and dynamics are demonstrated by example.

Consider Figures 1 and 2. This CP-net was constructed using Design/CPN, a tool in which types and inscriptions are written in a functional programming language called CPN ML. Types or colour sets may be arbitrarily complex and are defined on a declaration page associated with the CP-net. In Figure 1 the colour sets Roof, Chassis, Strut, Welder and Workers are enumeration types. The WorkerAndInfo, WorkerAndStrut, WorkerAndRoof, ChassisAndStrut and Car colour sets are comprised of tuples. Bay is a union of the colour sets Chassis, ChassisAndStrut and Car. roof, chassis, strut and car are typed variables. Functions can be defined as well: fun strut_type() takes a chassis as input and returns the strut that is required for that chassis. Similarly, fun roof_type() returns the type of roofing panel.

Consider the CP-net structure in Figure 2. A place may only contain tokens which be- 


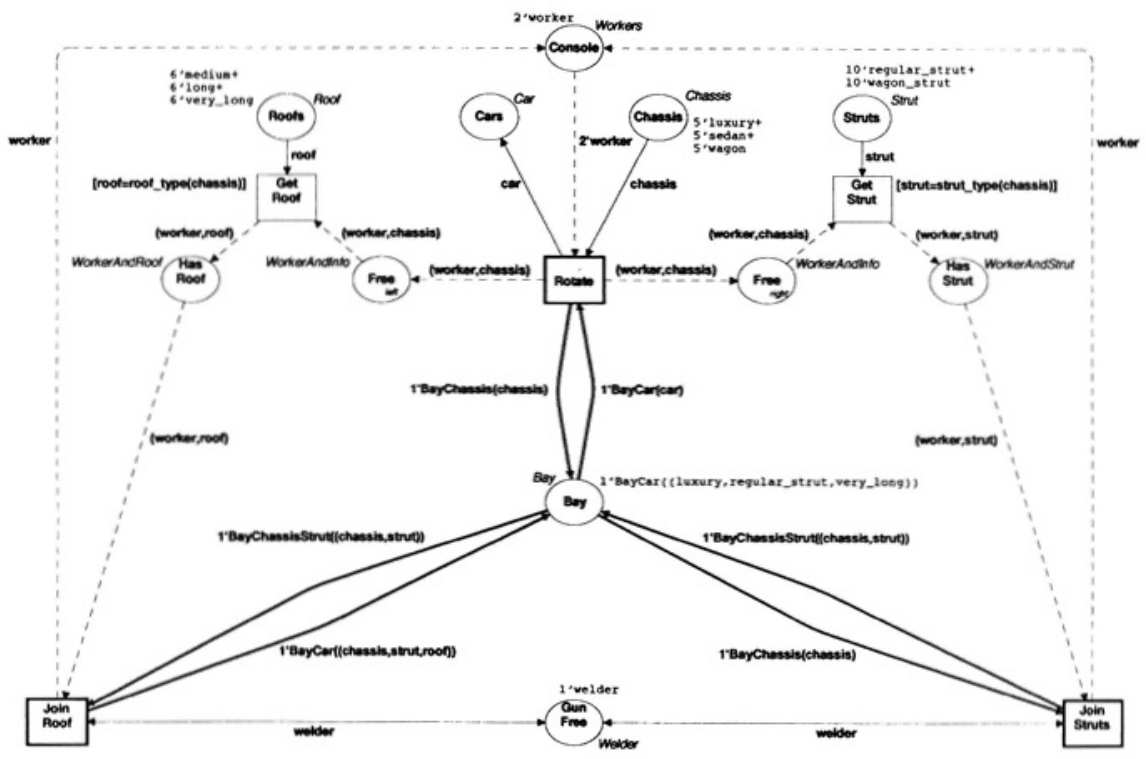

Figure 2 Top Level CP-net of the Assembly Line

long to its colour set. For example, the place Roofs has a colour set Roof and hence may only contain the tokens medium, long and very long. Adjacent to the place Chassis is the inscription ' 5 'luxury +5 'sedan +5 'wagon'. This is an initial marking (of a place). The inscription is a multi-set where multiple instances of a particular token are represented by a preceding integer and apostrophe. Arcs may be inscribed with expressions of arbitrary complexity (including functions) that evaluate to multi-sets (over the colour set of the associated place). A guard is a boolean expression associated with a transition. The expression must be true for the transition to occur. The transitions Get Roof and Get Strut are guarded (by the expressions in square brackets).

Execution of a CP-net is also best demonstrated by example. Consider the transition Rotate. Bay, Chassis and Console are input places and Bay, Cars and the two Free places are output places with respect to Rotate. The three input places have non-empty initial markings. For Rotate to be enabled there must be sufficient tokens on the input places to satisfy the input arc expressions. This is the case: two worker tokens are at the Console; a car token is in the Bay; and there are chassis tokens in the Chassis place (five instances of three colours). Variables car and chassis must be bound to tokens before Rotate is enabled. The bindings in Figure 3 are possible. Rotate occurs for one of these bindings. Assuming that the transition occurs with binding $b_{3}$, the resultant marking of output places Free left, Cars, Free ${ }_{\text {right }}$ and Bay are given in Figure 4. 


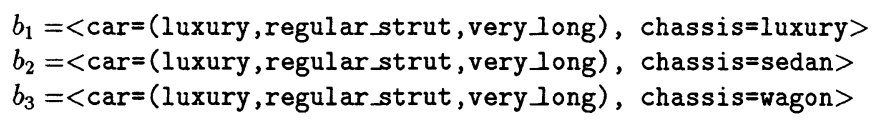

Figure 3 Possible Bindings for Rotate

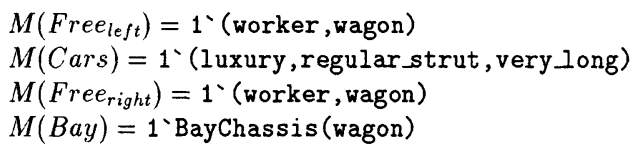

Figure 4 Rotate Occurred for $b_{3}$

\section{MODELLING THE ASSEMBLY LINE}

\subsection{Top-Down Modelling}

A top-down approach to modelling recognises that even the most simple models are of some benefit when it comes to understanding system behaviour and may be built upon as more detail is required.

Petri nets are well suited to the task. First a simple system is constructed with only the most basic or important characteristics and then simulated. Once satisfied with the net's behaviour, extra conditions or control structures may be added or transitions may be replaced with sub-nets until every significant element of the system is represented in the model. This technique has been well documented [Jensen, 1992, Zhou and Leu, 1991, Zhou et al., 1989, Desrochers and Al-Jaar, 1995].

It is useful to introduce some terminology at this point. Manufacturing systems make products. The market ready product which leaves a system differs from the raw products which enter because it has been processed by the agents which make up and are controlled by the system. This generic terminology groups workers with agents and worker behaviour with control systems.

More precisely then: processes are (collections of) manufacturing events which transform products (usually from a less evolved to a more evolved state); agents are the entities (physical or logical) which enable and perform processes (and so transform or transport products); control systems coordinate the agents.

Some extra terminology is needed to support the notion that a manufacturing system consists of many smaller systems (from factory, to assembly line, to workbay, $\cdots$, to clamp). Processes may be unresolved or fully resolved. An unresolved process is a high-level process (such as adding a roof) where control implementation details are not provided. An unresolved process may represent many other processing steps in a manufacturing system (e.g., the top-most unresolved process in automotive assembly is 'build car'). A fully resolved process is a machine-level (or worker-level) event which may not be resolved any further. The agents which enable these events are called control agents and correspond to controller or worker requirements (typically PLC output register values or worker behaviour). 


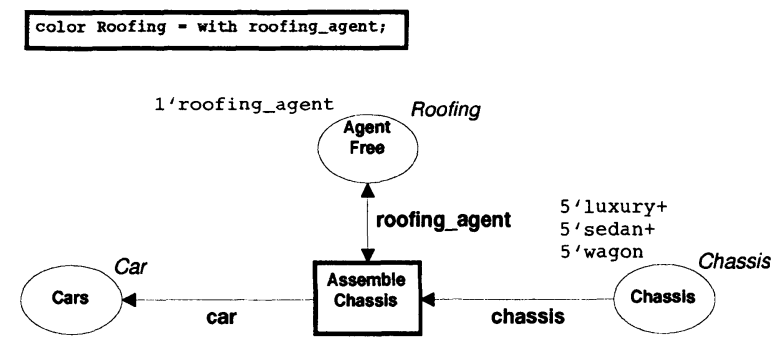

Figure 5 The Roofing Bay as a Single Transition

\subsection{Applying the Top-Down Approach to the Bay}

A full description of the methodology is given in [Farrington and Billington, 1996]. It may be summarised as follows: Places are assigned to each agent state and product location. A transition is assigned to each process. Agent states are made input places of those processes for which they are pre-conditions and output places of those processes for which they are post-conditions. If a transition represents a fully resolved or machine level process then the agent states preceding or proceeding the transition correspond to discrete control logic or machine-level events. Tokens are defined for each agent. Agent states are represented by tuples. Tokens are defined for each product. Product locations are represented by union colour sets. Arcs are then drawn linking pre-condition places to the respective processes, and processes to the respective post-conditions. Each unresolved process must now be resolved. Local products, processes and agents are again identified and a new CP-net structure built to reflect the extra resolution in the model.

The top level CP-net of the roofing bay consists of of a single transition representing the entire roofing process and places to represent the roofing bay agent and the input and output chassis buffers (see Figure 5). CP-nets are hierarchical. The transition Assemble Chassis in Figure 5 represents the sub-net in Figures 1 and 2. Consider now the unresolved process Join Roof in Figure 2. The top-down methodology has been used to construct a CP-net for Join Roof (see Figure 6). The processes (transitions) in Figure 6 are fully resolved (i.e., they are machine-level events) and so define the control logic that must be provided to enable the correct event sequencing, the actuators and sensors needed to communicate this information and the man machine interface (MMI) required to integrate the automatic and manual steps in the roofing bay.

Similarly for the other unresolved processes in the assembly line model, the top-down approach yields a set of control logic requirements. These requirements may be converted into PLC logic if needed. However, in this modelling exercise the requirements are not developed any further. Instead the existing control logic (PLC ladder logic) is directly modelled and developed from the bottom up. The bottom-up models are then fused with the top-down models so that extra system details can be incorporated into the (until now idealised) model of the assembly line. The final, fused model may then be analysed, executed, verified and form the basis for future modelling activities. 


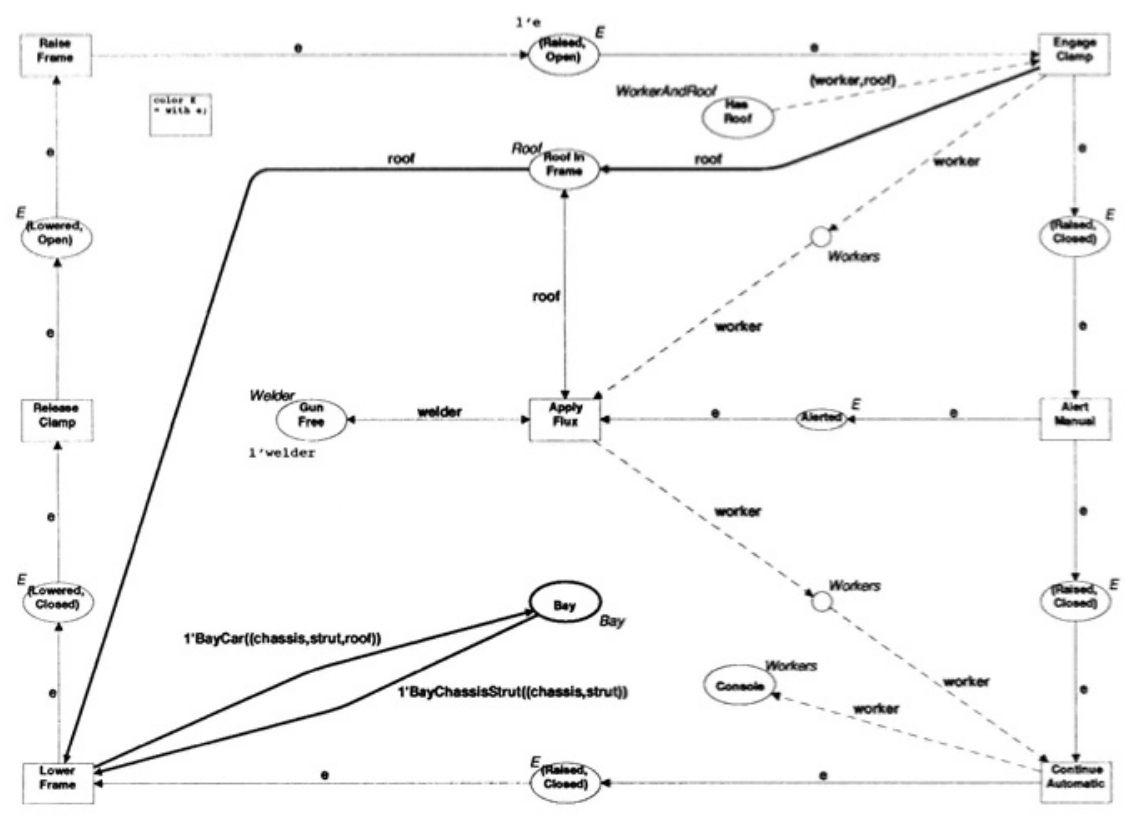

Figure 6 The Roofing Process Resolved

\subsection{Bottom-Up Modelling}

The translation of PLC ladder logic into Petri nets is already being automated, with some researchers proposing algorithms for the design of Petri nets in particular application areas [Venkatesh et al., 1994]. Such automated techniques produce ordinary Petri ncts (monochromatic CP-nets). These may then be collapsed into much smaller CPnets. The collapsing or colouring process recognises that large systems consist of subsystems with duplicate functionality. Large systems can therefore be modelled using a single CP-net structure and different coloured tokens for each sub-section rather than by many duplicate nets. Domain specific tools have appeared that automate this process [Darabi and Jafari, 1994].

In this paper a PLC architecture is modelled by CP-nets. Input, output and internal registers, the PLC program and, when needed, a representation of the discrete steps in a scan cycle are all included in the model. The control lines and sensor lines used to attach a PLC to the system may be described by state vectors and are modelled using lists. Hence the model is already collapsed a great deal compared with the approaches reported above.

The hardware which makes up the assembly line may be described in terms of machinelevel states and events. For example, a clamp is an agent which may be open or closed. The events which place the clamp in these states are release and engage respectively. A PLC-based feedback loop similar to the one shown in Figure 7 controls the assembly line. 


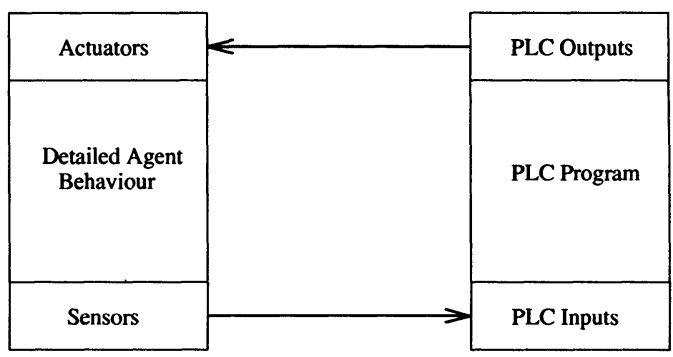

Figure 7 The Bottom-Up Model of the Assembly Line Control Loop

Many of the agents in the assembly line are connected to sensors. Sensors simply translate the state of an agent into the on-off input signals that are recognised by the PLC. A PLC program executes repeatedly, operating on the PLC inputs and a set of variables held inside the PLC. The program calculates a set of outputs, the states of which determine the operation of actuators which update the state of the agents in the assembly line. The state of the clamp, for example, is measured by a sensor connected to a PLC input. If the clamp is open the PLC input is switched on. If the clamp is closed the same PLC input is switched off. The clamp state is dependent on the actuator which switches it between states, and so is also dependent on the PLC output which drives the actuator.

The control loop is modelled by the Figures 8 and 9. In this generic model the agent behavioural dependencies are represented by the single transition Map Agents. The subnet associated with this transition defines the relationship between the elements in each agent. For example, the PLC controls the raising and lowering of the overhead frame by toggling a single switch. There is however no sensor which tests for the frame being in its correct lowered state - rather a proximity switch tests for the clamp being lowered. Hence, the Map Agent sub-net includes a transition which enforces the movement of the frame and clamp in unison - reflecting the fact that they are joined.

The CP-net in Figure 9 executes as follows: An initial token in the place Sensed Agents lists the initial states of the assembly line agents which are connected to sensors. The transition Set Sensors occurs, removing the token and placing a new token in Sensor Values. The new token (returned by the function sensor_op()) now represents the agent states in terms of PLC control signals. Scan Inputs occurs, copying this state information into the Input Registers of the PLC. The transition Execute Logic represents a sub-net which contains a CP-net model of the PLC ladder logic and performs the logic scanning function. Execute Logic occurs, removing the token from the place Input Registers and the initial token from the place Internal Registers. The two lists of register values are converted into a form which allows the Execute Logic sub-net to correctly simulate scanning behaviour. At the end of the scan the new output and internal register values are converted into lists and copied into the Output Registers and Internal Registers respectively. The transition Set Outputs then occurs. The output register values are converted into actuator switch settings (or switching requests) by the function set_switches(). The transition Switch Agent State then occurs, with the function set_agent() updating the states of those agents which have actuating circuits connected directly to the PLC outputs. The transition Map Agents then occurs, its 


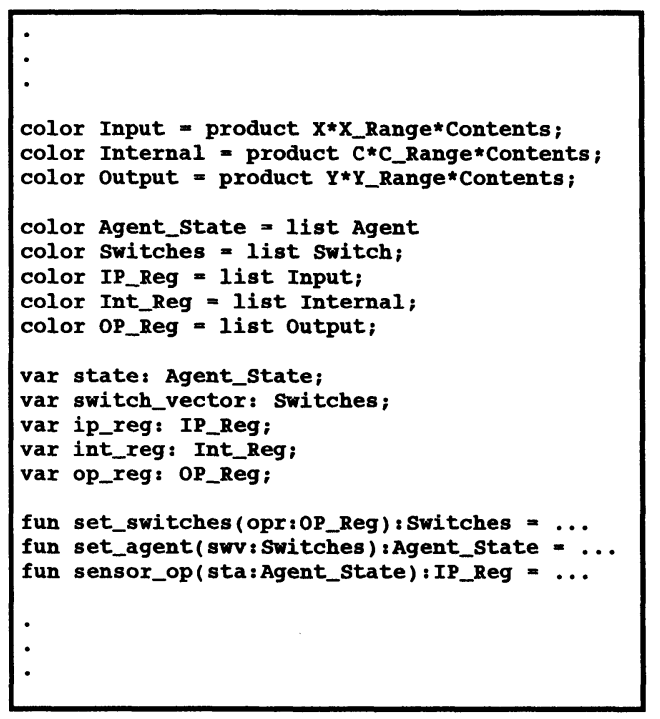

Figure 8 Global Declaration Page Fragment for the Control Loop

sub-net updating the states of any other components in the manufacturing system. The execution cycle then repeats.

\subsection{Fusing the two Models}

Figure 10 summarises the fusion process. Every sub-net which contains machine-level processes and control agents is replaced with a new, fused sub-net, which more closely resembles the CP-net in Figure 9. For example, in Figure 6 the places and transitions corresponding to the overhead frame agent (such as place (Lowered,Open) and transition Raise Frame) are replaced with a new representation of the frame based on the AGENT CP-net in Figure 9. The places and transitions in Figure 6 which correspond to product locations or worker agents are preserved and incorporated in the new model. Each fused AGENT CP-net is then connected to the single PLC CP-net which controls the entire bay (and the entire assembly line).

\section{CONCLUSIONS}

Top-down modelling techniques and CP-net tools have been used to develop control logic specifications for a PLC-based automotive assembly line. Here the system was modelled in terms of products, agents and processes. Bottom-up modelling techniques and CP-net tools have been used to construct a model of the PLC logic, the physical relationships between actuators and sensors and the control loop found in the assembly line.

The initial top-down modelling activity promoted system understanding while the 


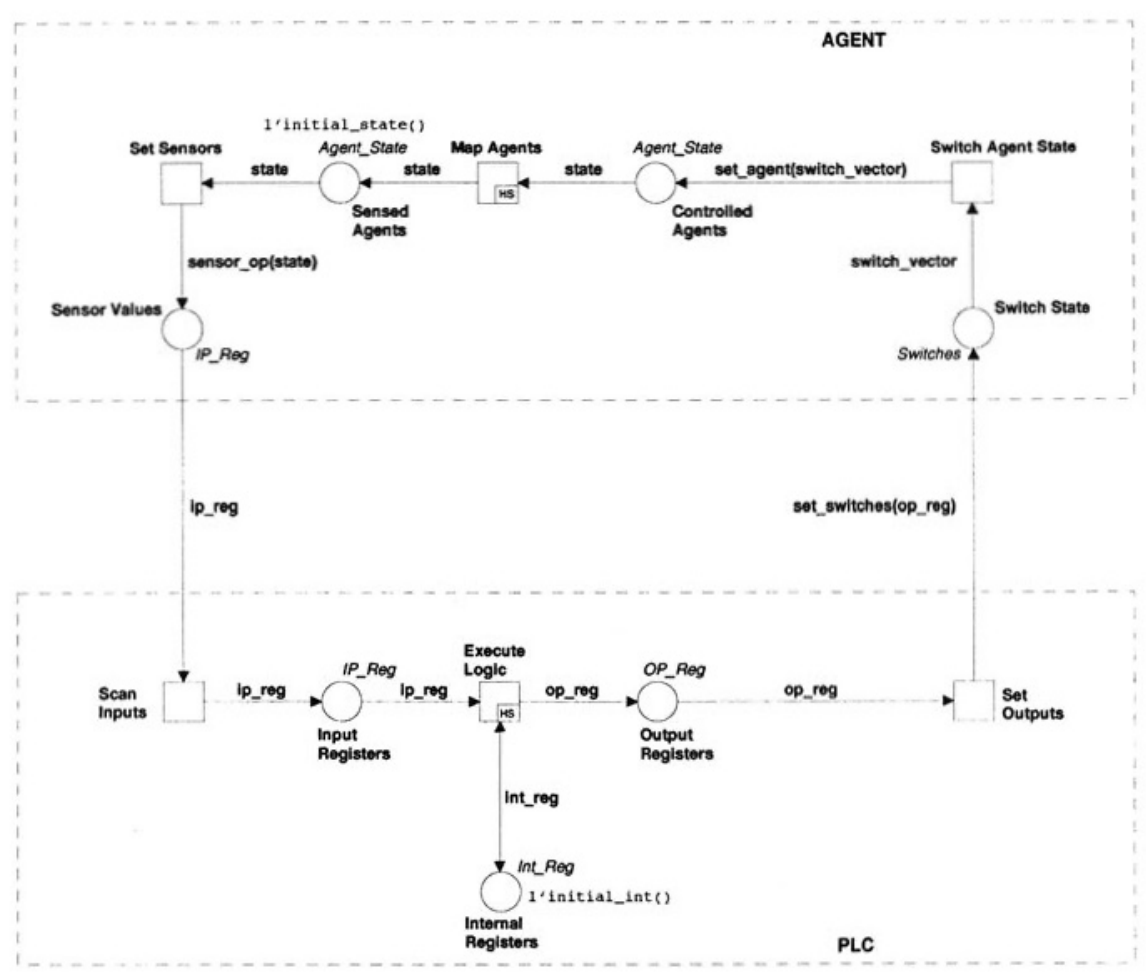

Figure 9 The Control Loop CP-net

bottom-up modelling made use of existing system control logic. The fusion process, summarised in Section 4.4, yields a system model which contains a hierarchy of behavioural requirements and a set of control logic specifications, so making it easier to identify the relationships between the bottom-level control logic specifications and the higher level system behavioural requirements. The fused CP-net model is therefore a useful starting point from which to derive an error-free assembly line control specification.

\section{REFERENCES}

[Billington, 1991] Billington, J. (1991). FORSEEing Quality Telecommunications Software. 1st Aust. Conf. on Telecommunications Software pp. 169-74.

[Darabi and Jafari, 1994] Darabi, H. and M.A. Jafari (1994). A Zero-One Programming of Petri Nets to Coloured Petri Net Transformation. Proc. 4th Int. Conf. on CIM and Automation Technology pp. 25-31.

[Desrochers and Al-Jaar, 1995] Desrochers, A.A. and R.Y. Al-Jaar (1995). Applications 


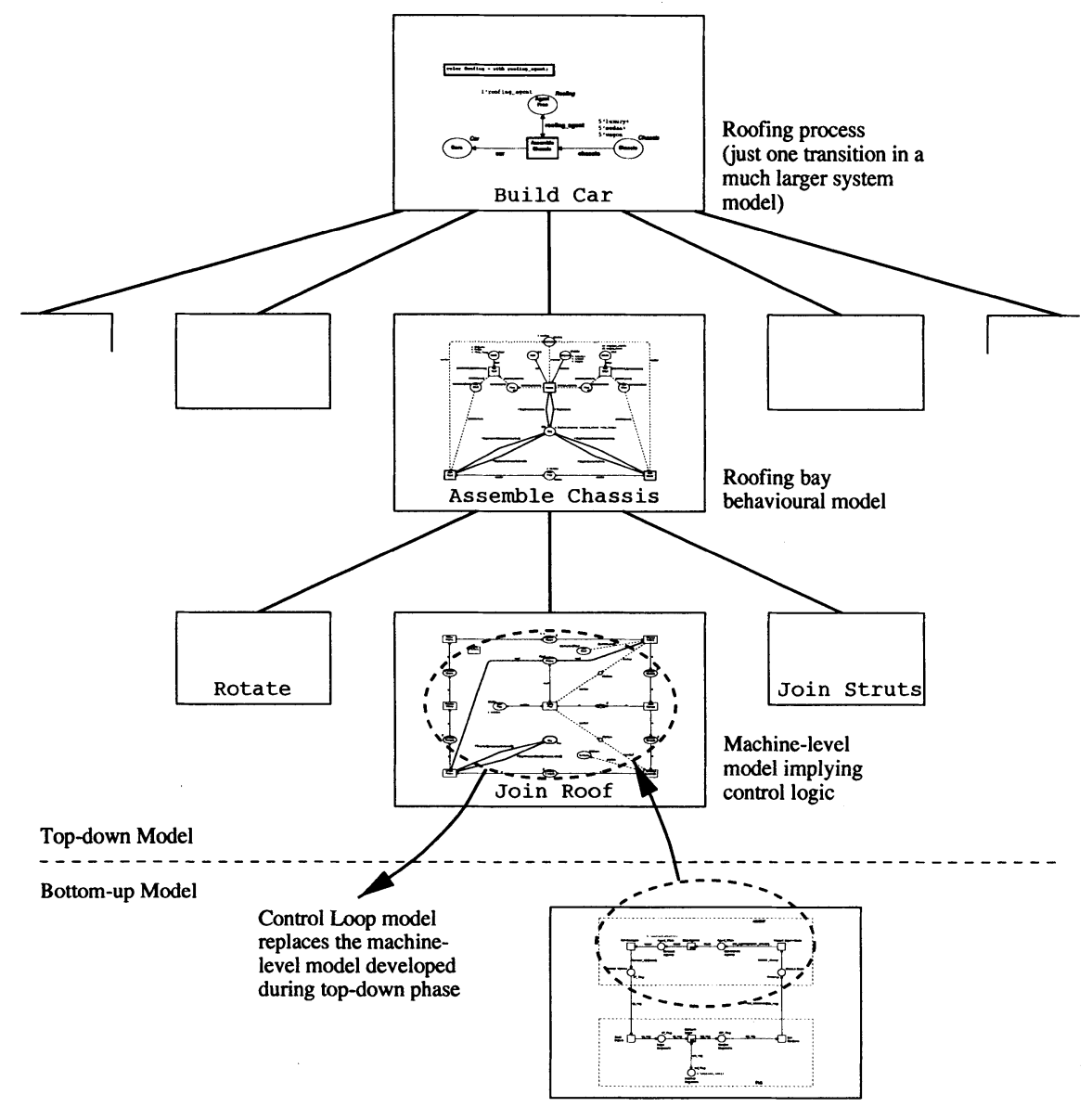

Figure 10 Fusing the Top-Down and Bottom-Up models

of Petri nets in manufacturing systems: modeling, control, and performance analysis. IEEE Press.

[Farrington and Billington, 1996] Farrington, M. and J. Billington (1996). Modelling an Automotive Assembly Line. Proc. 13th IFAC World Congress.

[Gilles, 1990] Gilles, M. (1990). Programmable Logic Controllers: Architecture and Applications. John Wiley \& Sons. pp. 1-20, 165-213.

[Jensen, 1992] Jensen, K. (1992). Coloured Petri Nets Basic Concepts, Analysis Methods and Practical Use. Springer-Verlag.

[Lester, 1994] Lester, L.N. (1994). TORAS Documentation. Final documentation for the TORAS development contract. UNICO Computer Systems.

[Reisig, 1992] Reisig, W. (1992). A Primer in Petri Net Design. Springer-Verlag. 
[Venkatesh et al., 1994] Venkatesh, K., M.C. Zhou and R. Caudill (1994). Automatic Generation of Petri Net Models from Logic Control Specifications. Proc. 4th Int. Conf. on CIM and Automation Technology pp. 242-247.

[Venkatesh, et al., 1994] Venkatesh, K., M.C. Zhou and R. Caudill (1994). Comparing Ladder Logic Diagrams and Petri Nets for Sequence Controller Design Through a Discrete Manufacturing System. IEEE Trans. on Industrial Electronics vol. 41, no. 6, pp. 611619.

[Zhou and Leu, 1991] Zhou, M.C. and M.C. Leu (1991). Petri net modeling of a flexible assembly station for printed circuit boards. Proc. IEEE Int. Conf. on Robotics and Automation pp. 2530-2535.

[Zhou et al., 1989] Zhou, M.C., F. DiCesare and A.A. Desrochers (1989). A top-down approach to systematic synthesis of petri net models for manufacturing systems. Proc. IEEE Int. Conf. on Robotics and Automation pp. 534-539. 